Available online on 15.06.2020 at http://ajprd.com
(C) 2013-20, publisher and licensee AJPRD, This is an Open Access article which permits unrestricted non-
commercial use, provided the original work is properly cited

Open Access

Review Article

\title{
A Technical Review: Solid- Lipid Nanoparticle (SLN), Their Characteristics and Their Preparation
}

\author{
Sunirmal Bhattacharjee1, Rabindra Debnath², Sinha Ashutosh Kumar ${ }^{3}$, Arpan Saha ${ }^{4}$, Surajit \\ Saha5, Sujata Debnath6.
}

Department of Pharmaceutics, Bharat Pharmaceutical Technology, Amtali, NH-8, Agartala, Tripura, India.

\begin{abstract}
A B S T R A C T
Solid lipid nanoparticles are at the forefront of the rapidly developing field of nanotechnology with several potential applications in drug delivery, clinical medicine and research as well as in other varied sciences. Due to their unique size-dependent properties, lipid nanoparticles offer the possibility to develop new therapeutics. The ability to incorporate drugs into nanocarriers offers a new prototype in drug delivery that could be used for secondary and tertiary levels of drug targeting. Hence, solid lipid nanoparticles hold great promise for reaching the goal of controlled and site specific drug delivery and hence have attracted wide attention of researchers. This review presents a broad treatment of solid lipid nanoparticles discussing their advantages, limitations and their possible remedies. The different types of nanocarriers which were based on solid lipid like solid lipid nanoparticles, nanostructured lipid carriers, lipid drug conjugates are discussed with their structural differences. Different production methods which are suitable for large scale production and applications of solid lipid nanoparticles are described. Appropriate analytical techniques for characterization of solid lipid nanoparticles like photon correlation spectroscopy, scanning electron microscopy, differential scanning calorimetry are highlighted. Aspects of solid lipid nanoparticles route of administration and their biodistribution are also incorporated. If appropriately investigated,solid lipid nanoparticles may open new vistas in therapy of complex diseases.
\end{abstract}

Keywords: Solid lipid nanoparticles, colloidal carriers, DLS, DSC, Homogenization, TEM, PCS.

A R T I C L E I N F O: Received 28 April 2020; Review Completed 06 June 2020; Accepted 10 June 2020; Available online 15 June. 2020
Cite this article as:

Sunirmal Bhattacharjee, Department of Pharmaceutics, Bharat Pharmaceutical Technology, Amtali, NH-8, Agartala, Tripura, India.

\section{INTRODUCTION:}

$\mathrm{C}$ olloidal particles ranging in size between 10 and $1000 \mathrm{~nm}$ are known as nanoparticles. They are manufactured from synthetic/natural polymers and ideally suited to optimize drug delivery and reduce toxicity. Over the years, they have emerged as a variable substitute to liposomes as drug carriers. The successful implementation of nanoparticles for drug delivery depends on their ability to penetrate through several anatomical barriers, sustained release of their contents and their stability in the nanometer size. However, the scarcity of safe polymers with regulatory approval and their high cost have limited the wide spread application of nanoparticles to clinical medicine ${ }^{1}$. To overcome these limitations of polymeric nanoparticles, lipids have been put forward as an alternative carrier, particularly for lipophilic pharmaceuticals. These lipid nanoparticles are known as solid lipid nanoparticles (SLNs), which are attracting wide attention of formulators world-wide ${ }^{2}$. SLNs are colloidal carriers developed in the last decade as an alternative system to the existing traditional carriers (emulsions, liposomes and polymeric nanoparticles). They are a new generation of submicron-sized lipid emulsions where the liquid lipid (oil) has been substituted by a solid lipid. SLN offer unique properties such as small size, large surface area, high drug loading and the interaction of phases at the 
interfaces, and are attractive for their potential to improve performance of pharmaceuticals, neutraceuticals and other materials ${ }^{3}$. SLNs are attracting major attention as novel colloidal drug carrier for intravenous applications ${ }^{1}$. The SLNs are sub-micron colloidal carrier which is composed of physiological lipid, dispersed in water or in an aqueous surfactant solution. The Pubmed search till the date indicates the trends in SLN research, given in fig. 1. So if systematically investigated, SLNs may open new vista in research and therapy ${ }^{4}$.

\section{ADVANTAGES}

- Use of biodegradable physiological lipids which decreases the danger of acute and chronic toxicity and avoidance

- of organic solvents in production method.

- Improved bioavailability of poor water soluble molecules.

- Site specific delivery of drugs, enhanced drug penetration into the skin via dermal application.

- Possibility of controlled drug release and drug targeting.

- Protection of chemically labile agents from degradation in the gut and sensitive molecules from outer environment.

- SLNs have better stability compared to liposomes.

- Enhance the bioavailability of entrapped bioactive and chemical production of labile incorporated compound.

- High concentration of functional compound achieved.

- Lyophilization possible ${ }^{2,5,25,31}$.

\section{Disadvantages of SLN}

- Poor drug loading capacity.

- Drug expulsion after polymeric transition during storage.

- Relatively high water content of the dispersions (7099.9\%).

- The low capacity to load water soluble drugs due to partitioning effects during the production process ${ }^{11}$

- Eccentric gelation propensity.

- Unforeseen motion of polymeric transition. ${ }^{12,15}$.

\section{Principle of Drug Release from SLN:}

The general standards of medication discharge from lipid nanoparticles are as per the following:

Higher surface territory because of little molecule measure in nanometer extent gives higher medication discharge.

Slow medication discharge can be accomplished when the medication is homogenously scattered in the lipid framework. It depends on sort and medication entanglement model of SLN.

Crystallinization conduct of the lipid carrier and high portability of the medication lead to quick medication discharge.

Fast initial drug release in the first $5 \mathrm{~min}$ in the drug enriched shell model as a result of the outer layer of particle due to larger surface area of drug depositon on the particle surface.
The burst release is reduced with increasing particle size and prolonged release could be obtained when the particles were sufficiently large, i.e., lipid macromolecules.

The type of surfactant and its concentration, which will interact with the outer shell and affect its structure, should be noted as the outer factor which is important, because a low surfactant concentration leads to a minimal burst and prolonged drug release.

The particle size affect drug release rate directly depends on various parameters such as composition of SLN formulation (such as surfactant, structural properties of lipid, drug) production method and conditions (such asproduction time, equipment, sterilization and lyophilization ${ }^{5}$.

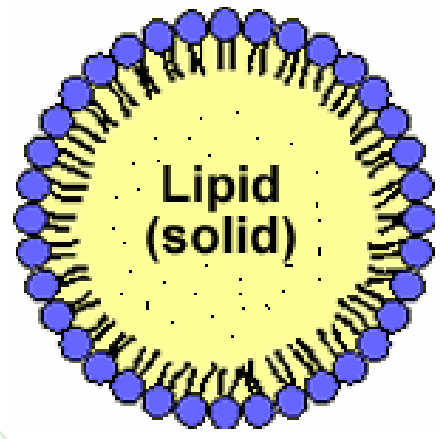

Figure: 1 Structure of solid lipid nanoparticle (SLN)

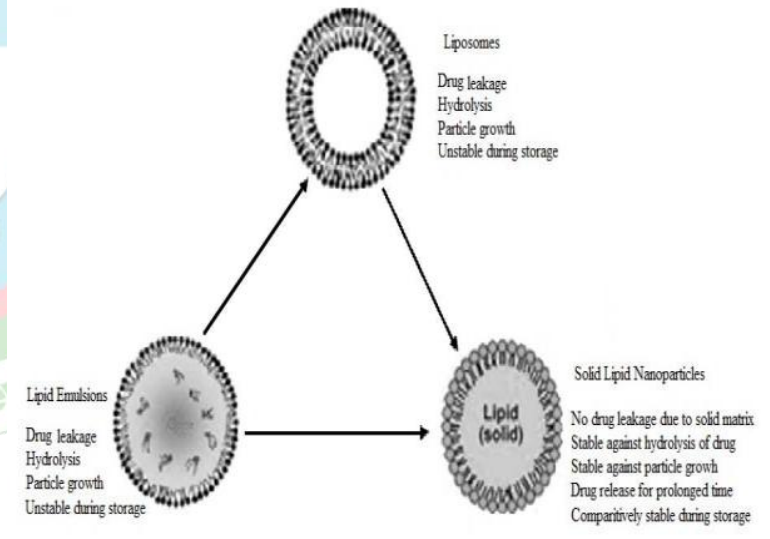

Figure: 2 A diagrammatic representation on SLN over emulsions and liposome's

Preparation of solid lipid nanoparticles 1- 4,6,43,52,56

The performance of SLNs greatly depends on the method of preparation which in turn influences the particle size,

drug loading capacity, drug release, drug stability etc. Different approaches exist for the production of finely

dispersed lipid nanoparticle dispersions. Few of the production processes such as high pressure homogenization and

microemulsion dilution have demonstrated scaling up possibility, a prerequisite for introduction of a product to the

market ${ }^{16,17}$. SLNs are prepared from lipid, emulsifier and water/solvent by using different methods and are discussed below. 


\section{Methods of preparation of solid lipid nanoparticles}

1. High pressure homogenization
A. Hot homogenization
B. Cold homogenization

2. Ultrasonication/high speed homogenization

1. Probe ultrasonication

2. Bath ultrasonication

3. Microemulsion based method

4. Supercritical fluid method

5. Spray drying method

6. Double emulsion method

7. Precipitation technique

8. Film-ultrasound dispersion.

9. Solvent evaporation method

\section{High shear homogenization:}

High shear homogenization technique were initially used for the production of solid lipid nanodispersions ${ }^{17,18}$. Both methods are widespread and easy to handle. However, dispersion quality is often compromised by the presence of micro particles. High-speed homogenization method is used to produce SLN by melt emulsification ${ }^{19}$. Olbrich et al. investigated the influence of different process parameters, including emulsification time, stirring rate and cooling condition on the particle size and zeta potential. Lipids used in this study included trimyristin, tripalmitin, a mixture of mono, di and triglycerides (Witepsol W35, Witepsol H35) with glycerol behenate and poloxamer 188 used as steric stabilizers $(0.5 \% \mathrm{w} / \mathrm{w})$.

\section{Hot homogenization:}

Hot homogenization is carried out at temperatures above the melting point of the lipid and can therefore be regarded as the homogenization of an emulsion. A pre-emulsion of the drug loaded lipid melt and the aqueous emulsifier phase (same temperature) is obtained by high-shear mixing device. HPH of the pre-emulsion is carried out at temperatures above the melting point of the lipid. In general, higher temperatures result in lower particle sizes due to the decreased viscosity of the inner phase.

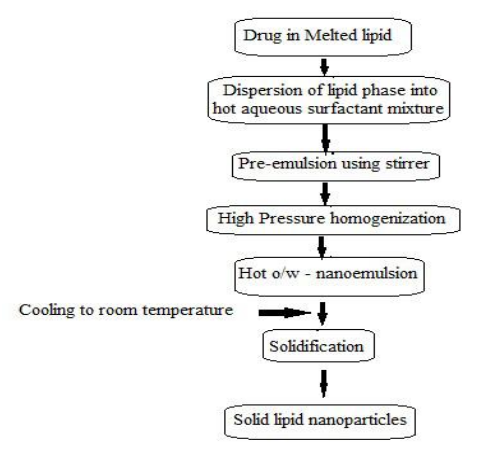

Figure. 4: Solid lipid nanoparticles preparation by hot homogenization process

\section{Cold homogenization}

Cold homogenization has been developed to overcome various problems associated with hot homogenization such as: Temperature-induced drug degradation, drug distribution into the aqueous phase during homogenization, Complexity of the crystallization step of the nanoemulsion leading to several modifications and/or super cooled melts. In this technique the drug containing lipid melt is cooled, the solid lipid ground to lipid microparticles and these lipid microparticles are dispersed in a cold surfactant solution Yielding a pre-suspension. Then this pre-suspension is homogenized at or below room temperature, the gravitation force is strong enough to break the lipid microparticles directly to solid lipid nanoparticles.

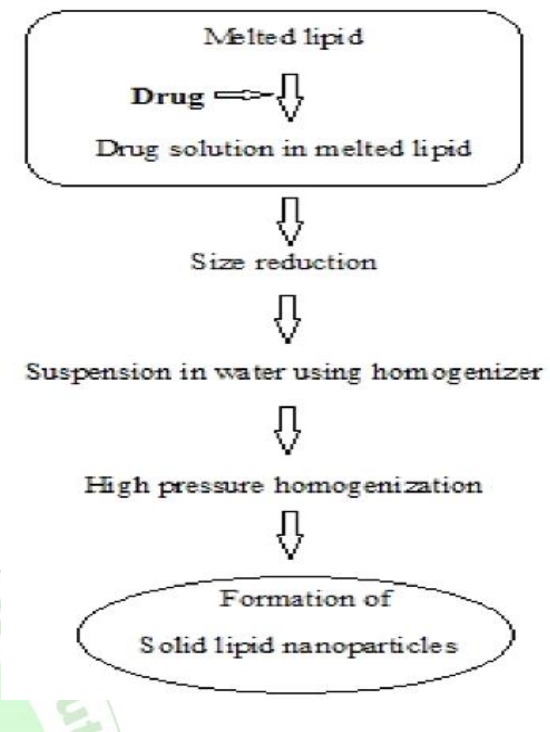

Figure: $\mathbf{5}$ Solid lipid nanoparticles preparation by cold homogenization process

\section{Ultrasonication/high speed homogenization}

For the production of nanoparticle dispersions by precipitation in $\mathrm{o} / \mathrm{w}$ emulsions ${ }^{25}$ the lipophilic material is dissolved in water-immiscible organic solvent (cyclohexane) that is emulsified in an aqueous phase. Upon evaporation of the solvent nanoparticle dispersion is formed by precipitation of the lipid in the aqueous medium. The mean diameter of the obtained particles was $25 \mathrm{~nm}$ with cholesterol acetate as model drug and lecithin/sodium glycocholate blend as emulsifier. The reproducibility of the result was confirmed by Siekmann and Westesen, who produced the cholesterol acetate nanoparticles of mean size $29 \mathrm{~nm}^{26}$.

\section{Micro emulsion based SLN preparations:}

Gasco and co-workers developed SLN preparation techniques which are based on the dilution of microemulsions ${ }^{27}$. They are made by stirring an optically transparent mixture at 65-700 which is typically composed of a low melting fatty acid (stearic acid), an emulsifier (polysorbate 20 , polysorbate 60 , soy phosphatidylcholine, and sodium taurodeoxycholate), co-emulsifiers (sodium monooctylphosphate) and water. The hot microemulsion is dispersed in cold water (2-30 ) under stirring. Typical volume ratios of the hot microemulsion to cold water are in the range of 1:25 to 1:50. The dilution process is critically determined by the composition of the microemulsion. 

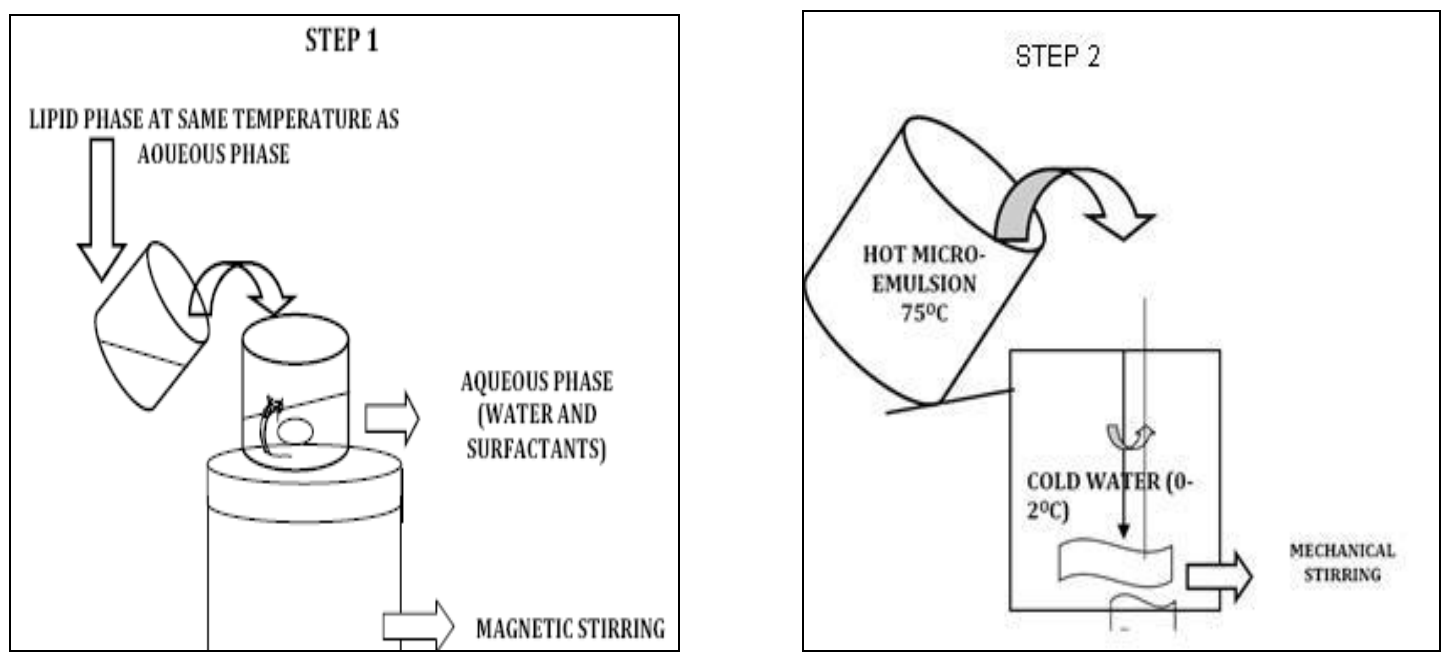

Figure: 6 Solid lipid nanoparticles preparation by micro emulsion method process

\section{SLN preparation by using supercritical fluid:}

This is a relatively new technique for SLN production and has the advantage of solvent-less processing ${ }^{32,33}$. There are several variations in this platform technology for powder and nanoparticle preparation. SLN can be prepared by the rapid expansion of supercritical carbon dioxide solutions (RESS) method. Carbon dioxide (99.99\%) was the good choice as a solvent for this method ${ }^{34}$.

\section{Spray drying method:}

It's an alternative procedure to lyophilization in order to transform an aqueous SLN dispersion into a drug product. It's a cheaper method than lyophilization. This method cause particle aggregation due to high temperature, shear forces and partial melting of the particle. Freitas and Mullera ${ }^{35}$ recommends the use of lipid with melting point $>700$ for spray drying. The best result was obtained with SLN concentration of $1 \%$ in a solution of trehalose in water or $20 \%$ trehalose in ethanol-water mixtures $(10 / 90 \mathrm{v} / \mathrm{v})$.

\section{Double emulsion method}

Here the drug is encapsulated with a stabilizer to prevent the partitioning of drug in to external water phase during solvent evaporation in the external water phase of $\mathrm{w} / \mathrm{o} / \mathrm{w}$ double emulsion. For the preparation of hydrophilic loaded SLN, a novel method based on solvent emulsificationevaporation has been used ${ }^{36}$. Here the drug is encapsulated with a stabilizer to prevent drug partitioning to external water phase during solvent evaporation in the external water phase of $\mathrm{w} / \mathrm{o} / \mathrm{w}$ double emulsion.

\section{Precipitation method}

The glycerides are dissolved in an organic solvent (e.g. chloroform) and the solution will be

emulsified in an aqueous phase. After evaporation of the organic solvent the lipid will be precipitated forming nanoparticles.

\section{Film-ultrasound dispersion:}

The lipid and the drug were put into suitable organic solutions, after decompression, rotation and

evaporation of the organic solutions, a lipid film is formed, then the aqueous solution which includes theemulsions was added. Using the ultrasound with the probe to diffuser at last, the SLN with the little and uniform particle size is formed.

\section{Solvent evaporation}

SLNs can also prepared by solvent evaporation method. The lipophilic material is dissolved in a

Water-immiscible organic solvent (e.g. cyclohexane) that is emulsified in an aqueous phase. Upon evaporation of the solvent, nanoparticles dispersion is formed by precipitation of the lipid in the aqueous medium by giving the nanoparticles of $25 \mathrm{~nm}$ mean size. The solution was emulsified in an aqueous phase by high pressure homogenization. The organic solvent was removed from the emulsion by evaporation under reduced pressure (40-60 mbar).

\section{CONCLUSION}

Solid lipid nanoparticles do not, as proposed, "combine the advantages of other colloidal drug carriers and avoid the disadvantages of them". The results cannot simply be regarded as nanoemulsions with a solid core. Clear advantages of SLN include the composition (physiological compounds), the rapid and effective production process including the possibility of large scale production, the avoidance of organic solvents and the possibility to produce carriers with higher encapsulation efficiency. Disadvantages include low drug-loading capacities, the presence of alternative colloidal structures (micelles, liposomes, mixed micelles, drug nanocrystals), the complexity of the physical state of the lipid (transformation between different modifications) and the possibility of super cooled melts which cause stability problems during storage or administration (gelation, particle size increase, drug expulsion). Sample dilution or water removal might significantly change the equilibria between the different colloidal species and the physical state of the lipid. The appropriate characterization of the complex surfactant/lipid dispersions requires several analytical methods in addition to the determination of the particle size.

\section{REFERENCES;}

1. Lee WW. Chewing gum as a delivery vehicle for pharmaceutical and neutraceuticals substances. Pharm Tech Online 2001; 2:1-11. 
2. Edgar W, Geddes D. Chewing gum and dental health - A review. Br Dent J 1990; 168:173-177.

3. Conway B. Chewing gum as a drug delivery system. The Drug Delivery Companies Report Autumn/Winter; 2003:33-35.

4. Patel PV, Desai TR, Dedakiya AS, Bandhiya HM. Medicated chewing gum: A review. IJUPLS 2011; 1(1):111-128.

5. Biswal PK and Kumar A. An Updated Review on Medicated Chewing Gum. IJAPBC 2013; 2(2):351-59.

6. Jacobsen J, Christrup LL, Jensen NH. Medicated chewing gum. Am J Drug Deliv 2004; 2:75-88.

7. Cloys L, Christen A, Christen J. The development \& history of chewing gum. Bulletin of the History of Dentistry 1992; 40:57-65.

8. Owens, L. Gumtech to Produce Aspergum and Chooz for ScheringPlough Health Care Products. Business Wire 1998.

9. Khanekar P, Mhatre S, Momin M. Medicated Chewing Gum: A Potential Drug Delivery System. IJPFR 2012; 2(4):64-75.

10. Morjaria Y, Irwin WJ, Barnett PX, Chanvv RS, Conway BR. In vitro Release of nicotine from chewing gum formulations. Dissolution Technologies 2004; 12-15.

11. Naik H, Gupta S. Medicated Chewing Gums- Updated Review. IJPRD 2011; 2 (8):66-76.

12. Jacobsen J, Christrup LL, Jensen NH. Medicated chewing gum: Pros and Cons. Am J Drug Deliv 2004; 2(2):75-88.

13. oldberg LD, Ditchek NT. Chewing gum diarrhea, Am J Dig Dis 1978; 23(6):568-62.
14. Addy M, Roberts WR. Comparison of the bis iguanid antiseptics alexandrine and chlorhexidine II. Clinical and in vitro staining properties. J Clin. Periodontol 1981; 8(3):220-30.

15. Munksgaard EC, Nolte J, Kristensen K. Adherence of chewing gum to dental restorative materials. Am J Dent 1995; 8(3):137-139.

16. Weil AT. Coca leaf as a therapeutic agent. Am J Drug Alcohol Abuse 1978; 5(1):75-86.

17. Martin R. Chewing gum. BMJ 1985; 290:1232-1236.

18. Edgar WM, Dawes C, O'Mullane D. Saliva and oral health: An essential overview for the health professional. 3rd ed., British Dental Association Publication, London; 2004.

19. Sharma NS, Arjariya PA, Jat RC, Fiza F, Sharma G, Rathore SA, Tiwari R. An overview on medicated chewing gum and its applications. IJPFR 2013; 4(3):3158-73.

20. Zyck DJ, Greenberg MJ, Barkla DG, Marske SW, Schnell PG, Mazzone P.. Method of making coated chewing gum products containing various antacids. US Patent 2003; 6:635-45.

21. Kahtani D. Chewing gum: trick or treat.SDJ 1999; 11(1):27-34.

22. Athanikar NK, Gubler SA. Process for manufacturing a pharmaceutical chewing gum. US Patent 2001; 6:322-828.

23. Subraman CR, Kirshnayya B. Tableted chewing gum composition and method of preparation. US Patent 1988; 4:753-805

24. Keizo M, Yokomichi FY. Process for the preparation of chewing gum. US Patent 1976; 4:321. 